\title{
Deflationary $\Lambda(t)$ cosmology: Observational expressions
}

\author{
J. V. Cunha, J. A. S. Lima, and N. Pires \\ Departamento de Física, Universidade Federal do Rio Grande do Norte, CP 1641, 59072-970 Natal, RN, Brasil \\ Received 23 January 2002 / Accepted 15 May 2002

\begin{abstract}
We discuss the classical cosmological tests for a large class of FRW type models driven by a decaying vacuum energy density. Analytic expressions for the lookback time, age of the universe, luminosity distance, angular diameter, and galaxy number counts versus redshift are derived and their meaning discussed in detail. It is found that the standard cold dark matter $(\mathrm{CDM})$ results are significantly altered, showing that such tests may constrain appreciably the physical parameters of these models which are also in agreement with the accelerated expansion suggested by the latest SNe type Ia observations. Cosmology: decaying vacuum, kinematic tests
\end{abstract}

Key words. cosmology: observations - cosmology: theory - cosmology: distance scale

\section{Introduction}

Recent measurements from some type Ia Supernovae (SNe) at intermediate and high redshifts (Perlmutter et al. 1999; Riess et al. 1998) indicate that the bulk of energy in the Universe is repulsive and appears like a "quintessence" component, that is, an unknown form of dark energy (in addition to the ordinary CDM matter) probably of primordial origin (see Turner 2000 for a review). Together with the observations of CMB anisotropies (de Bernardis 2000), such results seem to provide an important piece of information connecting an early inflationary stage with the astronomical observations.

This state of affairs has stimulated the interest in more general models containing an extra component describing this dark energy, and simultaneously accounting for the present accelerated stage of the Universe. However, the absence of convincing evidence on the nature of the dark component gave origin to an intense debate and mainly to theoretical speculations. A possible list of old and new candidates for "quintessence" now include:

(i) a decaying vacuum energy density, or a time varying $\Lambda$-term (Özer \& Taha 1987; Freese et al. 1987; Chen \& Wu 1990; Carvalho et al. 1992; Waga 1993; for reviews see Overduin \& Cooperstoock 1998; Sahni \& Starobinski 2000);

(ii) the so-called "X-matter", an extra component simply characterized by an equation of state $p_{x}=\omega \rho_{x}$, where $\omega \geq-1$ (Turner \& White 1997; Chiba et al. 1997; Efstathiou 1999; Lima \& Alcaniz 2000; Turner 2000; Alcaniz \& Lima 2001), which describes, as a particular case, cosmologies with a constant $\Lambda$-term ( $\Lambda$ CDM models). Generically, the $\omega$ parameter may be a function of the redshift (Cooray \& Huterer 2000);

Send offprint requests to: J. A. S. Lima, e-mail: limajas@dfte.ufrn.br (iii) a rolling scalar field (Ratra \& Peebles 1988; Caldwell et al. 1998; Wang et al. 2000).

Here we are interested in the first class of models. The basic reason is the widespread belief that the early Universe evolved through some phase transitions, thereby yielding a vacuum energy density which at present is at least 118 orders of magnitude smaller than in the Planck time (Weinberg 1989). Such a discrepancy between theoretical expectation (from the modern microscopic theory of particles and gravity) and empirical observations constitutes a fundamental problem in the interface uniting astrophysics, particle physics and cosmology, which is often called "the cosmological constant problem" (Weinberg 1989; Jack NG 1992; Dolgov 1997). This puzzle inspired some authors (Lima \& Maia 1994; Lima \& Trodden 1996) to propose a class of phenomenological deflationary cosmologies driven by a decaying vacuum energy density where the present value, $\Lambda_{\mathrm{o}}=\Lambda\left(t_{\mathrm{o}}\right)$, is a remnant of the primordial inflationary stage (from now on the subscript "o" denotes the present day quantities). The basic scenario has an interesting cosmological history that evolves in three stages. Initially, an unstable de Sitter configuration, with no matter and radiation is supported by the largest values of the vacuum energy density. This nonsingular de Sitter state evolves to a vacuum/radiation dominated phase, and, subsequently, the Universe changes continuously from vacuum/radiation to the present vacuum/dust dominated phase. The first stage harmonizes the scenario with the cosmological constant problem, while the transition to the second stage solves the horizon and other well-know problems in the same manner as in inflation. Finally, the Universe enters in the present vacuum/dust phase with a negative deceleration parameter as required by the $\mathrm{SNe}$ type Ia observations.

Another interesting feature of $\Lambda(t)$ models is that the temperature dependence on the redshift $z$ of the relic radiation, $T(z)$, can be slightly different from the standard prediction 
deduced from the adiabatic expansion. Actually, indirect measurements of $T(z)$ at high redshifts (Songaila et al. 1994; Ge et al. 1997) may become one of the most powerful cosmological tests because it may exclude the presence of a cosmological constant or even any kind of separately conserved quintessence (Lima et al. 2000).

In this article, we focus our attention on this class of deflationary decaying vacuum models. The effective time dependent cosmological term is regarded as a second fluid component with energy density, $\rho_{\mathrm{v}}(t)=\Lambda(t) / 8 \pi G$, which transfers energy continuously to the material component. The main goal is to investigate the basic kinematic tests in the present vacuum/dust dominated phase, or equivalently, how the classical cosmological tests may constrain the physical parameters of such models. The paper is organized as follows: in Sect. 2, we set up the basic equations for deflationary cosmologies driven by a decaying $\Lambda(t)$-term. In Sect. 3 , the expressions for classical cosmological tests are derived and compared with the conventional expressions without the $\Lambda$-term. Section 4 gives the conclusion of the main results, and, in the Appendix A, the exact expression yielding the dimensionless radial coordinate as a function of the redshift is deduced.

\section{Deflationary $\Lambda(t)$ cosmology: Basic equations}

We shall consider a class of spacetimes described by the general Friedmann-Robertson-Walker (FRW) line element $(c=1)$

$\mathrm{d} s^{2}=\mathrm{d} t^{2}-R^{2}(t)\left(\frac{\mathrm{d} r^{2}}{1-k r^{2}}+r^{2} \mathrm{~d} \theta^{2}+r^{2} \sin ^{2} \theta \mathrm{d} \phi^{2}\right)$,

where $R(t)$ is the scale factor, $k=0, \pm 1$ is the curvature parameter of the spatial sections, and $r, \theta$ and $\phi$ are dimensionless comoving coordinates. In that background, the Einstein field equations (EFE) with a nonvacuum component plus a cosmological $\Lambda(t)$-term are:

$$
\begin{aligned}
& 8 \pi G \rho+\Lambda(t)=3 \frac{\dot{R}^{2}}{R^{2}}+3 \frac{k}{R^{2}}, \\
& 8 \pi G p-\Lambda(t)=-2 \frac{\ddot{R}}{R}-\frac{\dot{R}^{2}}{R^{2}}-\frac{k}{R^{2}},
\end{aligned}
$$

where an overdot means time derivative, $\rho$ and $p$ are the energy density and pressure, respectively. As usual, we consider that the nonvacuum component obeys the " $\gamma$-law" equation of state

$p=(\gamma-1) \rho$,

where $\gamma \in[1,2]$ specifies if the fluid component is radiation $\left(\gamma=\frac{4}{3}\right)$ or dust $(\gamma=1)$.

Phenomenologically, we also assume that the effective $\Lambda(t)$-term is a variable dynamic degree of freedom so that in an expanding universe it relaxes to its present value according with the following ansatz (Lima \& Maia 1994; Lima \& Trodden 1996)

$\rho_{\mathrm{v}}=\frac{\Lambda(t)}{8 \pi G}=\beta \rho_{\mathrm{T}}\left(1+\frac{1-\beta}{\beta} \frac{H}{H_{\mathrm{I}}}\right)$,

where $\rho_{\mathrm{v}}$ is the vacuum density, $\rho_{\mathrm{T}}=\rho_{\mathrm{v}}+\rho$ is the total energy density, $H=\dot{R} / R$ is the Hubble parameter, $H_{\mathrm{I}}^{-1}$ is the arbitrary time scale characterizing the deflationary period, and $\beta \in[0,1]$ is a dimensioneless parameter of order unity.

It is worth noticing that for $H=H_{\mathrm{I}}$, the above definition reduces to $\rho_{\mathrm{v}}=\rho_{\mathrm{T}}$ so that we have inflation with no matterradiation component $(\rho=0)$. In particular, if $H_{\mathrm{I}}^{-1}$ is the order of the Planck time, that is, $H_{\mathrm{I}}^{-1} \sim 10^{-43} \mathrm{~s}$, one may show that such models are in accordance with the cosmological constant problem in the sense that $\Lambda_{\text {Planck }} / \Lambda_{\mathrm{o}} \sim 10^{118}$.

At late times $\left(H \ll H_{\mathrm{I}}\right)$, we see from (5) that $\rho_{\mathrm{V}} \sim \beta \rho_{\mathrm{T}}$, as required by the recent Supernovae observations. To be more precise, if the deflationary process begins at Planck time, the ratio $H_{0} / H_{\mathrm{I}} \sim 10^{-60}$ while the remaining terms are of order unity. Indeed, even if deflation begins much later, say at $10^{-35} \mathrm{~s}$, or even at $10^{-15} \mathrm{~s}$ (the respective scales of grand and electroweak unification in the standard model), one obtains $H_{0} / H_{\mathrm{I}} \sim 10^{-52}$ and $H_{0} / H_{\mathrm{I}} \sim 10^{-32}$, respectively. This means that to a high degree of accuracy, the scale $H_{\mathrm{I}}$ is unimportant during the vacuum/dust dominated phase. Therefore, since in this work we mainly interested in the classical cosmological tests, henceforth we consider only this limiting behavior with the material component described by a pressureless fluid $(\gamma=1)$.

Let us now consider the evolution of the scale factor. Combining Eqs. (2)-(5) and taking the limit $H \ll H_{\mathrm{I}}$, it is readily seen that the scale factor during the vacuum/dust phase obeys the slightly modified FRW equation

$2 R \ddot{R}+(1-3 \beta) \dot{R}^{2}+(1-3 \beta) k=0$,

the first integral of which is

$\dot{R}^{2}=\frac{A}{R^{1-3 \beta}}-k$,

where the constant $A>0$ in order that $\rho$ be positive definite in this phase (see Eq. (2)). By expressing the constant $A$ in terms of the present day parameters, it is straightforward to show that the above equation can be rewritten as

$\left(\frac{\dot{R}}{R_{\mathrm{o}}}\right)^{2}=H_{\mathrm{o}}^{2}\left[1-\left(\frac{\Omega_{\mathrm{o}}}{1-\beta}\right)+\left(\frac{\Omega_{\mathrm{o}}}{1-\beta}\right)\left(\frac{R_{\mathrm{o}}}{R}\right)^{1-3 \beta}\right]$,

where $\left.\Omega_{\mathrm{o}} \equiv \frac{\rho}{\rho_{\mathrm{c}}}\right|_{t=t_{\mathrm{o}}}$ is the present value of the matter density parameter. For $\beta=0$ the above equation reproduces the standard cold dark matter result (Kolb \& Turner 1990). Whereas for $\beta \neq 0$, it describes the influence of the decaying vacuum in the present phase.

Following standard lines we also define the deceleration parameter $q_{\mathrm{o}}=-\left.\frac{R \ddot{R}}{\vec{R}^{2}}\right|_{t=t_{0}}$. Using Eqs. (6) and (7) one may show that

$q_{\mathrm{o}}=\frac{1-3 \beta}{2}\left(\frac{\Omega_{\mathrm{o}}}{1-\beta}\right)$

Then, for any value of $\Omega_{\mathrm{o}} \neq 0$, we see that the deceleration parameter $q_{\mathrm{o}}$ with decaying vacuum energy is always smaller than the corresponding one of the CDM model. The critical case $\left(\beta=\frac{1}{3}, q_{\mathrm{o}}=0\right)$ describes a "coasting cosmology". However, instead of being supported by "K-matter" (Kolb 1989), this kind of model is obtained in the present context for a vacuum/dust filled universe, and the corresponding solutions hold regardless of the value of $\Omega_{0}$. It is also interesting that even 


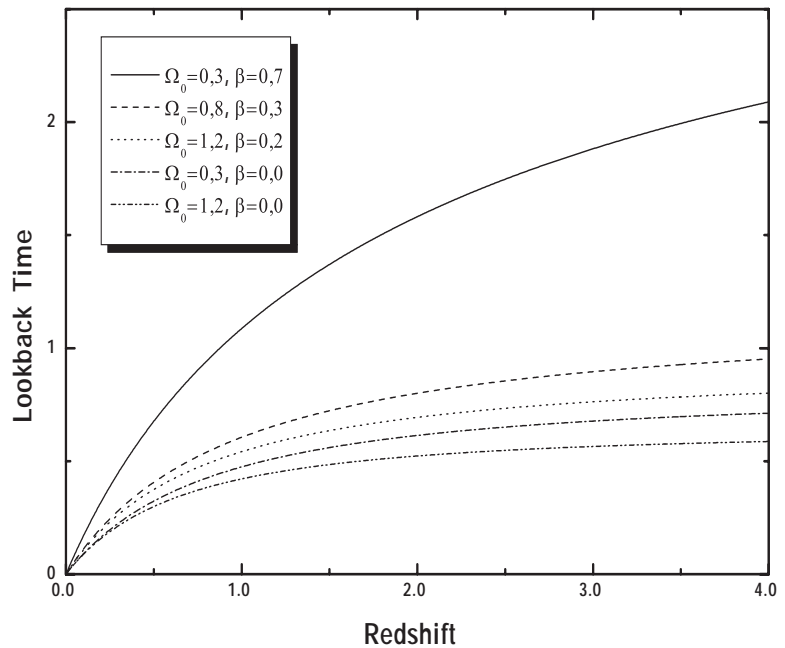

Fig. 1. Lookback time as a function of the redshift for some selected values of $\Omega_{0}$ and $\beta$. The models with larger vacuum energy rate $\beta$ are older.

negative values of $q_{\mathrm{o}}$ are allowed since the constraint $q_{\mathrm{o}}<0$ can always be satisfied provided $\beta>1 / 3$. These results are in line with recent measurements of the deceleration parameter $q_{\mathrm{o}}$ using Type Ia supernovae (Perlmutter et al. 1999; Riess et al. 1998). Such observations indicate that the universe may be accelerating today, which corresponds dynamically to a negative pressure term in the EFE. For a fixed $\Omega_{0}$, this means that the universe with decaying vacuum is older than the corresponding FRW model with the usual deceleration parameter $q_{\mathrm{o}} \geq 0$. This behavior also reconcile other recent results (Freedman 1998), pointing to a Hubble parameter $H_{\mathrm{o}}$ larger than $50 \mathrm{~km} \mathrm{~s}^{-1} \mathrm{Mpc}^{-1}$.

Now, combining Eqs. (2), (5) and (7) for $\left(H \ll H_{\mathrm{I}}\right)$ one finds that vacuum and the matter energy density can be expressed as

$\rho_{v}=\beta \rho_{\mathrm{T}}=\beta \rho_{\mathrm{T} 0}\left(\frac{R_{0}}{R}\right)^{3(1-\beta)}$,

$\rho=(1-\beta) \rho_{\mathrm{T}}=(1-\beta) \rho_{\mathrm{T} 0}\left(\frac{R_{0}}{R}\right)^{3(1-\beta)}$,

where $\rho_{\mathrm{T} 0}=3 A / 8 \pi G R_{0}^{3(1-\beta)}$. In virtue of the decaying vacuum energy, we also see that the explicit dependence of the energy density on the scale factor $R(t)$ is slightly modified in comparison with the standard case. As should be expected from Eqs. (6) and (7), some dynamic expressions may be obtained from the standard CDM ones simply by replacing the "index" $\gamma=1$ by an effective parameter $\gamma_{\mathrm{eff}}=1-\beta$ (see the above scale laws).

\section{Kinematic tests}

The kinematical relation distances must be compared with the observations in order to put limits on the free parameters of the deflationary class of models presented in the last section. As remarked before, $H_{\mathrm{I}}$ cannot be constrained by the classical tests since it is unimportant in the present phase. It is adjusted in order to put the models in accordance to the cosmological constant problem (Lima \& Trodden 1996). It thus follows

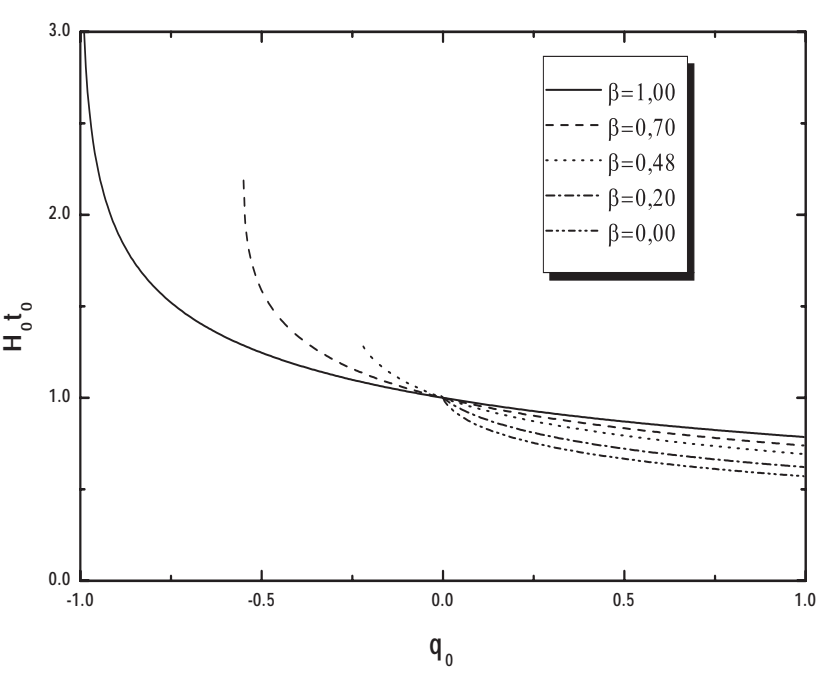

Fig. 2. Age parameter as a function of the deceleration parameter for some selected values of $\beta$.

that we have only the pair of free parameters $\left(\Omega_{0}, \beta\right)$ satisfying $\Omega_{\mathrm{T} 0}=\Omega_{0} /(1-\beta)$, where $\Omega_{\mathrm{T} 0}$ is the present value of the total density parameter.

\section{a) Lookback time-redshift diagram}

The lookback time, $\Delta t=t_{\mathrm{o}}-t(z)$, is the difference between the age of the universe at the present time $(z=0)$ and the age of the universe when a particular light ray at redshift $z$ was emitted. By integrating (8) such a quantity is easily derived

$$
t_{\mathrm{o}}-t(z)=H_{\mathrm{o}}^{-1} \int_{\frac{1}{1+z}}^{1} \frac{\mathrm{d} x}{\sqrt{1-\left(\frac{\Omega_{\mathrm{o}}}{1-\beta}\right)+\left(\frac{\Omega_{\mathrm{o}}}{1-\beta}\right) x^{-(1-3 \beta)}}}
$$

which generalizes the standard CDM result (Kolb \& Turner 1990). The age of the universe is obtained by taking the limit $z \rightarrow \infty$ in the above equation. We find

$t_{\mathrm{o}}=H_{\mathrm{o}}{ }^{-1} \int_{0}^{1} \frac{\mathrm{d} x}{\sqrt{1-\left(\frac{\Omega_{\mathrm{o}}}{1-\beta}\right)+\left(\frac{\Omega_{\mathrm{o}}}{1-\beta}\right) x^{-(1-3 \beta)}}}$.

For $\beta=0$ the above expressions reduce to the ones of the dustfilled FRW models (Kolb \& Turner 1990). Generically, we see that decaying vacuum increases the dimensionless parameter $H_{\mathrm{o}} t_{\mathrm{o}}$ while preserving the overall expanding CDM behavior. The lookback time curves as a function of the redshift for some selected values of $\Omega_{0}$ and $\beta$ are displayed in Fig. 1. For completeness, in Fig. 2 we show the age of the Universe (in units of $H_{\mathrm{o}}$ ) as a function of $q_{\mathrm{o}}$ for some selected values of $\beta$.

\section{b) Luminosity distance-redshift}

The luminosity distance of a light source is defined as the ratio of the detected energy flux $L$, and the apparent luminosity, i.e., $d_{L}^{2}=\frac{L}{4 \pi l}$. In the homogeneous and isotropic FRW metric (1) it takes the form below (Sandage 1988)

$d_{L}=R_{\mathrm{o}} r_{1}(z)(1+z)$ 


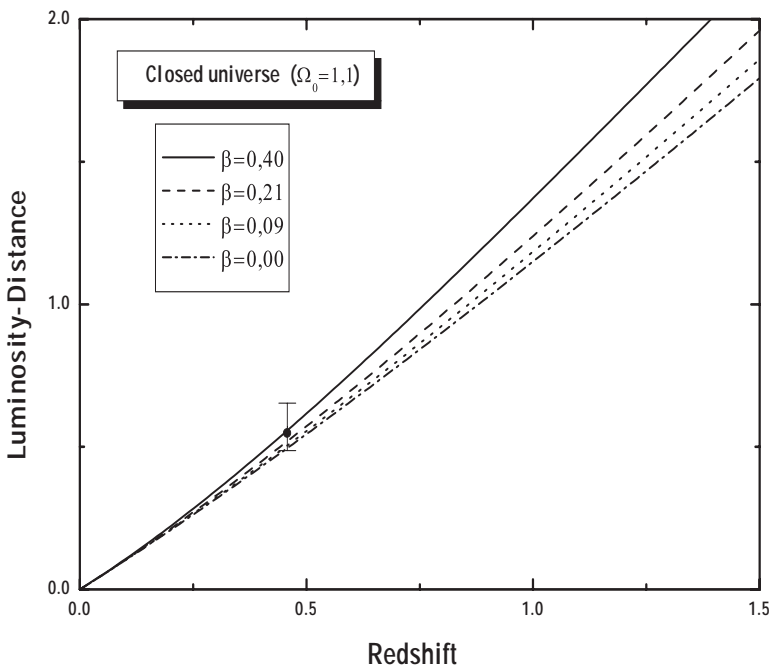

Fig. 3. Luminosity distance as a function of the redshift for closed models with decaying vacuum energy. Here and in Fig. 4 the typical error bar and data point are taken from Perlmutter et al. (1995).

where $r_{1}(z)$ is the radial coordinate distance of the object at light emission. Inserting $r_{1}(z)$ derived in the Appendix, it follows that

$d_{L}=\frac{(1+z) \sin \left[\delta \sin ^{-1}\left(\alpha_{1}\right)-\delta \sin ^{-1}\left(\alpha_{2}\right)\right]}{H_{\mathrm{o}}\left(\frac{\Omega_{\mathrm{o}}}{1-\beta}-1\right)^{\frac{1}{2}}}$

where $\delta=\frac{2}{(1-3 \beta)}, \alpha_{1}=\left(1-\frac{1-\beta}{\Omega_{o}}\right)^{\frac{1}{2}}$ and $\alpha_{2}=\alpha_{1}(1+z)^{-\left(\frac{1-3 \beta}{2}\right)}$.

As one may check, expressing $\Omega_{\mathrm{o}}$ in terms of $q_{\mathrm{o}}$ from (9), and taking the limit $\beta \rightarrow 0$, the above expression reduces to

$d_{L}=\frac{1}{H_{\mathrm{o}} q_{\mathrm{o}}^{2}}\left[z q_{\mathrm{o}}+\left(q_{\mathrm{o}}-1\right)\left(\sqrt{2 q_{\mathrm{o}} z+1}-1\right)\right]$,

which is the usual FRW result (Weinberg 1972). Expanding Eq. (15) for small $z$ gives

$H_{\mathrm{o}} d_{L}=z+\frac{1}{2}\left[1-\frac{1-3 \beta}{2}\left(\frac{\Omega_{\mathrm{o}}}{1-\beta}\right)\right] z^{2}+\ldots$,

which depends explicitly on the $\beta$ parameter. However, replacing $\Omega_{\mathrm{o}}$ from (9) we recover the usual FRW expansion for small redshifts, which depends only on the effective deceleration parameter $q_{\mathrm{o}}$ (Weinberg 1972). This is not a surprising result since expanding $d_{L}(z)$ in terms of $\Omega_{0}$, the " 00 " component of Einstein's equations has implicitly been considered, while the expansion in terms of $q_{\mathrm{o}}$ comes only from the form of the FRW line element. The luminosity distance as a function of the redshift for closed and open models with decaying vacuum energy is shown in Figs. 3 and 4, respectively. As expected for all kinematic tests, different cosmological models have similar behavior at $z \ll 1$, and the greatest discrimination among them comes from observations at large redshifts. A more quantitive analysis based on the SNe type Ia observations will be presented elsewhere.

\section{c) Angular size-redshift}

Another important kinematic test is the angular size - redshift relation $\theta(z)$. As widely known, the data concerning the

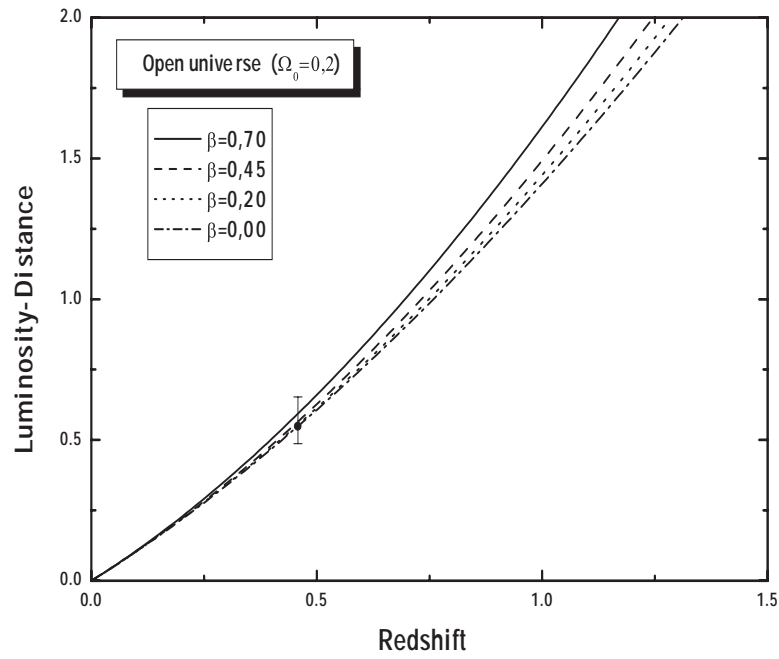

Fig. 4. Luminosity distance versus redshift: the case. Solid curve is the prediction for the standard open CDM universe $(\beta=0)$.

angular-size are still somewhat controversial (see Buchalter et al. 1998 and references therein). Here we are interested in angular diameters of light sources described as rigid rods and not as isophotal diameters. These quantities are naturally different, because in an expanding world the surface brightness varies with the distance (Sandage 1988). The angular size of a light source of proper size $D$ (assumed free of evolutionary effects) located at $r=r_{1}(z)$ and observed at $r=0$ is

$\theta=\frac{D(1+z)}{R_{\mathrm{o}} r_{1}(z)}$

Inserting the expression of $r_{1}(z)$ given in the Appendix $\mathrm{A}$ it follows that

$\theta=\frac{D H_{\mathrm{o}}\left(\frac{\Omega_{\mathrm{o}}}{1-\beta}-1\right)^{\frac{1}{2}}(1+z)}{\sin \left[\delta \sin ^{-1} \alpha_{1}-\delta \sin ^{-1} \alpha_{2}\right]}$.

For small $z$ one finds

$\theta=\frac{D H_{\mathrm{o}}}{z}\left[1+\frac{1}{2}\left(3+\frac{1-3 \beta}{2}\left(\frac{\Omega_{\mathrm{o}}}{1-\beta}\right)\right) z+\ldots\right]$.

Hence, decaying vacuum cosmologies as modeled here also requires an angular size decreasing as the inverse of the redshift for small $z$. However, for a given value of $\Omega_{\mathrm{o}}$, the second order term is a function only of the $\beta$ parameter. In terms of $q_{\mathrm{o}}$, inserting (9) into (20) it is readily obtained

$\theta=\frac{D H_{\mathrm{o}}}{z}\left[1+\frac{1}{2}\left(3+q_{\mathrm{o}}\right) z+\ldots\right]$

which is formally the same FRW result for small redshifts (Sandage 1988). At this limit only the effective deceleration parameter may be constrained from the data, or equivalently, at small redshifts one cannot extract the values of $\Omega_{\mathrm{o}}$ and $\beta$ separately. The angular size-redshift diagram for closed and open models and selected values of the $\beta$ parameter is displayed in Figs. 5 and 6, respectively. 


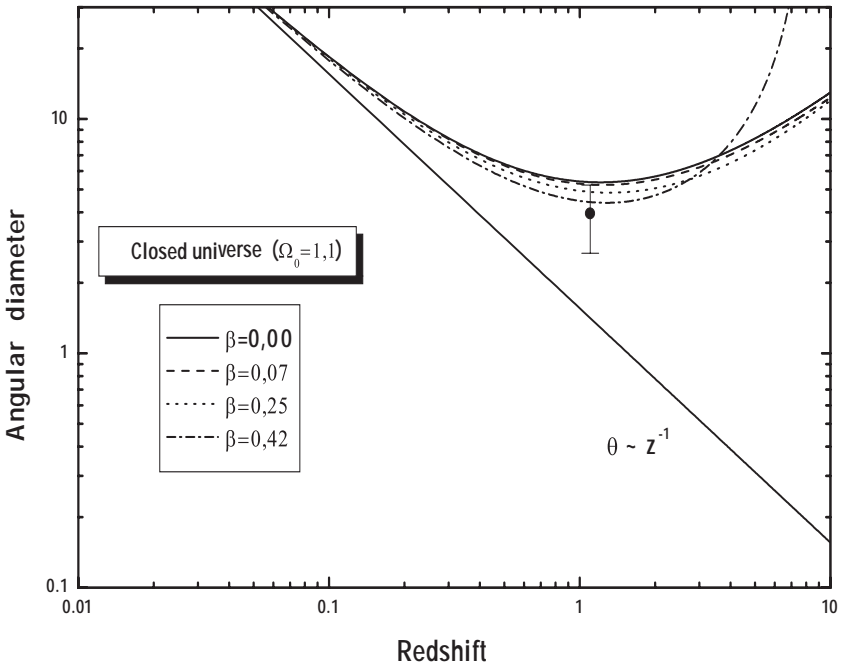

Fig. 5. Angular diameter versus redshift in closed models with decaying vacuum energy and some selected values of $\beta$. The minimum is the displaced Euclidian result. Here and in Fig. 6, the typical error bar and data point are taken from Gurvits et al. (1999).

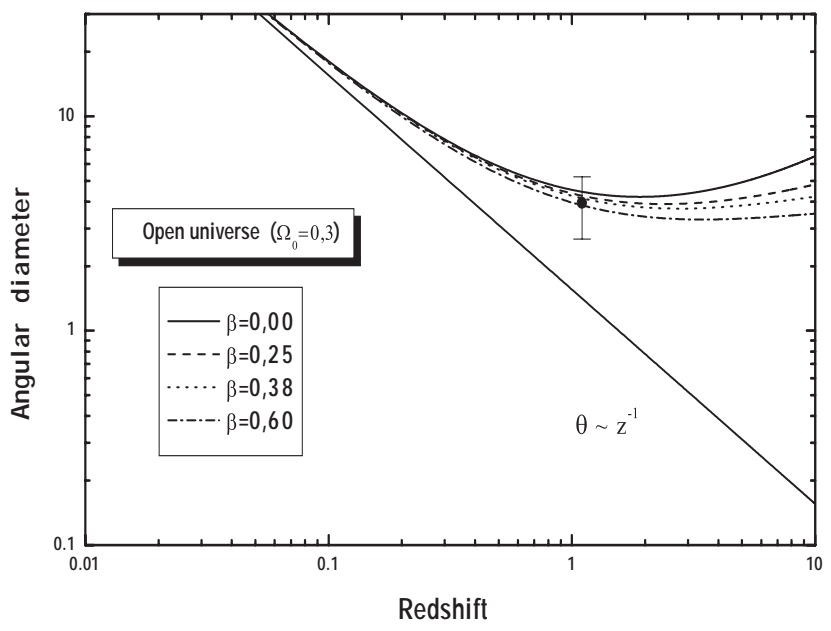

Fig. 6. The same graph of Fig. 5 for open model.

\section{d) Number counts-redshift}

The final kinematic test considered here is the galaxy number count per redshift interval. The number of galaxies in a comoving volume is equal to the number density of galaxies (per comoving volume) $n_{\mathrm{g}}$, times the comoving volume element $\mathrm{d} V_{\mathrm{c}}$

$\mathrm{d} N_{\mathrm{g}}(z)=n_{\mathrm{g}} \mathrm{d} V_{\mathrm{c}}=\frac{n_{\mathrm{g}} r^{2} \mathrm{~d} r \mathrm{~d} \Omega}{\sqrt{1-k r^{2}}}$.

By using $r_{1}(z)$ as derived in appendix, it follows that the general expression for number-counts can be written as

$\frac{\left(H_{\mathrm{o}} R_{\mathrm{o}}\right)^{3} \mathrm{~d} N_{\mathrm{g}}}{n_{\mathrm{g}} z^{2} \mathrm{~d} z \mathrm{~d} \Omega}=\frac{\sin ^{2} \delta\left[\sin ^{-1}\left(\alpha_{1}\right)-\sin ^{-1}\left(\alpha_{2}\right)\right]}{(1+z) z^{2} f\left(\Omega_{\mathrm{o}}, \beta, z\right)}$,

where $f\left(\Omega_{\mathrm{o}}, \beta, z\right)=\left(\frac{\Omega_{\mathrm{o}}}{1-\beta}-1\right)\left[1-\frac{\Omega_{\mathrm{o}}}{1-\beta}+\frac{\Omega_{\mathrm{o}}}{1-\beta}(1+z)^{1-3 \beta}\right]^{1 / 2}$. For small redshifts

$\frac{\left(H_{\mathrm{o}} R_{\mathrm{o}}\right)^{3} \mathrm{~d} N_{\mathrm{g}}}{n_{\mathrm{g}} z^{2} \mathrm{~d} z \mathrm{~d} \Omega}=1-2\left[\frac{\left(\frac{\Omega_{\mathrm{o}}}{1-\beta}\right)(1-3 \beta)}{2}+1\right] z+\ldots$

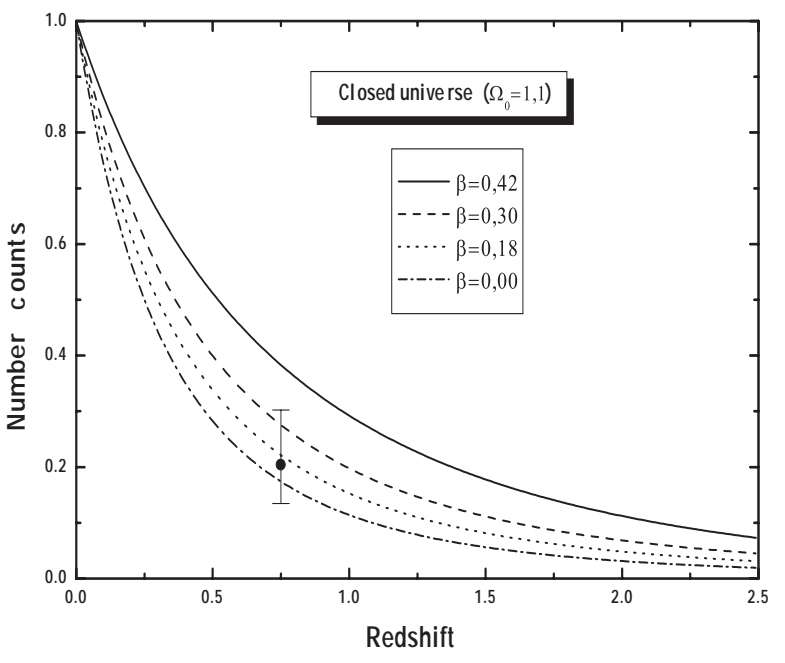

Fig. 7. Number counts as a function of the redshift for closed models with decaying vacuum. Here and in Fig. 8, the typical error bar and data point are taken from Loh \& Spillar (1986).

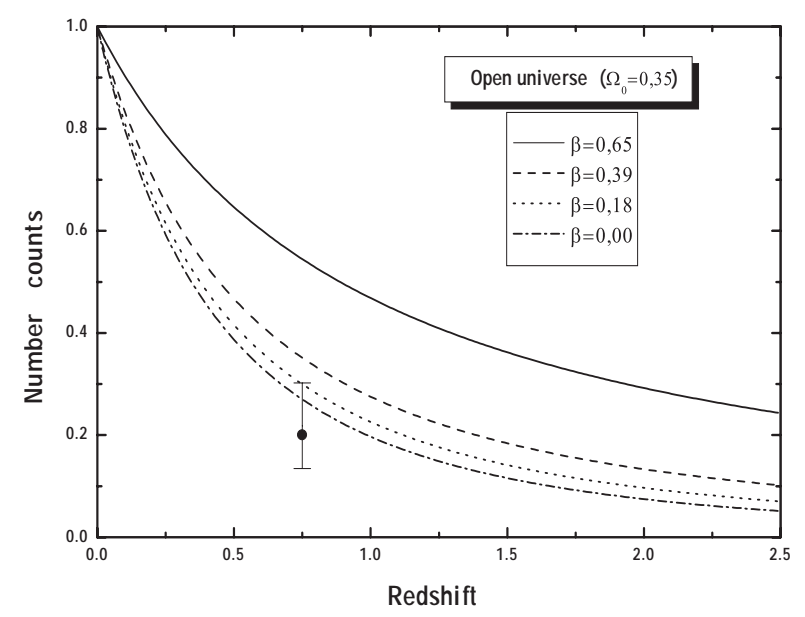

Fig. 8. Number counts versus redshift: the open case.

Table 1. Limits to $\beta$.

\begin{tabular}{crr}
\hline \hline Test & Open & Closed \\
\hline Luminosity distance-redshift & $\beta \leq 0.83$ & $\beta \leq 0.58$ \\
Angular size-redshift & $\beta \leq 0.69$ & $\beta \leq 0.56$ \\
Number counts-redshift & $\beta \leq 0.19$ & $\beta \leq 0.34$ \\
\hline
\end{tabular}

In Figs. 7 and 8 we have displayed the number counts-redshift relations for flat, closed and open Universes for some selected values of $\Omega_{\mathrm{o}}$ and $\beta$. It is worth mentioning the tendency of decaying vacuum models to have larger volumes per redshift interval than the standard FRW models with the same $\Omega_{\mathrm{o}}$. This feature is similar to the one found in "quintessence" and $\Lambda \mathrm{CDM}$ cosmologies and could be advantageous if the observational data indicate an excess count of high-redshift objects. The limits on the $\beta$ parameter obtained from all kinematic tests are shown in Table 1.

At this point, it is also interesting to compare our results with previous studies of kinematic tests for $\Lambda(t)$ cosmologies. 
Observable expressions for a large class of flat decaying vacuum models were discussed by Waga (1993). In his paper, the time varying cosmological term was defined by $\Lambda=\alpha R^{-2}+$ $\beta H^{2}+\gamma$, where $\alpha, \beta$ and $\gamma$ are arbitrary constants. Although rather different from the deflationary ansatz assumed here, we see that in the limit $H \ll H_{\mathrm{I}}$, our expression (5) reduces to $\rho_{\mathrm{v}} \sim \beta \rho_{\mathrm{T}}$, or equivalently, $\Lambda \sim \beta\left(3 H^{2}+3 k R^{-2}\right)$. Therefore, by considering only the flat case $(k=0)$ and taking $\alpha=\gamma=0$, the late stages of the deflationary cosmologies has exactly the same behavior of the models examined by Waga. Note also that our $\beta$ parameter must be identified with $1 / 3$ of that one appearing in his paper. More recently, some aspects involving the angular size, luminosity distance, and SNe type Ia observations in $\Lambda(t)$ models were also investigated by Vishwakarma (2000, 2001). By generalizing earlier papers (Chen \& Wu 1990; Carvalho et al. 1992), He considered the interesting ansatz $\Lambda=n \Omega H^{2}$, where $n$ is a dimensionless constant and $\Omega$ is the density parameter of the fluid component. Although proposed as a new cosmology, if we rewrite this $\Lambda(t)$ in terms of the total energy density, this decaying law corresponds precisely to the late stages of the deflationary universes for $\beta=\frac{n}{n+3}$. A more quantitative analysis accounting for angular sizes, the existence of old high redshifts galaxies, and ages constraints from globular clusters in this framework will be discussed in a forthcoming communication. We also remark that models driven by a gravitational adiabatic matter creation process (Calvão et al. 1992; Lima \& Alcaniz 1999; Alcaniz \& Lima 1999) present some kinematic expressions, like the dimensionless radial coordinate, which are similar to the ones discussed here for $\Lambda(t)$ cosmologies (see Appendix). In these models, the present day matter creation rate, $\psi_{\mathrm{o}}=3 n_{\mathrm{o}} H_{\mathrm{o}} \approx 10^{-16}$ nucleons $\mathrm{cm}^{-3} \mathrm{yr}^{-1}$, is also nearly the same rate transferred from the decaying vacuum to the fluid component. However, these two spacetimes are completely different from a physical viewpoint because the negative creation pressure of the former scenario cannot be interpreted as a genuine decaying vacuum component.

\section{Conclusion}

The recent observational evidence for an accelerated state of the present universe, obtained from distant SNe Ia (Perlmutter et al. 1999; Riess et al. 1998), gave strong support to the search for alternative cosmologies. As demonstrated here, a variable $\Lambda$-term or a decaying vacuum energy density is also an ingredient accounting for this unexpected observational result. In the present paper we have analyzed all kinematic expressions for flat, closed, and open cosmologies when the decaying vacuum is a fraction of the total energy density. At the later stages of the evolution, the rather slight changes introduced by $\Lambda(t)$, which is quantified by the $\beta$ parameter, provide a reasonable fit of several cosmological data points. Kinematic tests like luminosity distance, angular diameter and number-counts versus redshift relations constrain perceptively the decaying vacuum (see Table 1$)$. For models characterized by the pair $\left(\Omega_{0}, \beta\right)$, the age of the universe is always greater than the corresponding CDM model $(\beta=0)$, and even values bigger than $H_{\mathrm{o}}^{-1}$ are allowed for all values of the curvature parameter.

\section{Appendix A: Dimensionless radial coordinate versus redshift relation}

Some observable quantities in the standard FRW model are easily determined expressing the radial dimensionless coordinate $r$ of a source light as a function of the redshift (Mattig 1958). In this appendix, we derive a similar equation to the decaying vacuum energy scenario discussed in this paper.

Now consider a typical galaxy located at $\left(r_{1}, \theta_{1}, \phi_{1}\right)$ emitting radiation to an observer at $\left(r=0, \theta_{1}, \phi_{1}\right)$. If the waves leave the source at time $t_{1}$ and reach the observer at time $t_{0}$, the null geodesic equation defining the light track yields

$\int_{t_{0}}^{t_{1}} \frac{\mathrm{d} t}{R(t)}=\int_{0}^{r_{1}} \frac{\mathrm{d} r}{\sqrt{1-k r^{2}}}=\frac{\sin ^{-1} \sqrt{k} r_{1}}{\sqrt{k}}=I$.

Since $t=t(R)$, changing variable to $x=\frac{R}{R_{0}}$ and using (8), the above result reads

$I=\frac{1}{R_{\mathrm{o}} H_{\mathrm{o}}} \int_{\frac{1}{1+2}}^{1} \frac{\mathrm{d} x}{x \sqrt{1-\left(\frac{\Omega_{0}}{1-\beta}\right)+\left(\frac{\Omega_{\mathrm{o}}}{1-\beta}\right) x^{-(1-3 \beta)}}}$.

This integral depends on the values of the $\Omega_{\mathrm{o}}$ and $\beta$ parameters. For $\beta=\frac{1}{3}$ one finds the same results of the coasting cosmology (Kolb 1989). For $\beta$ different of $\frac{1}{3}$, we introduce a new auxiliary variable $y^{2}=\left[1-\left(\frac{1-\beta}{\Omega_{0}}\right)\right] x^{(1-3 \beta)}$, in terms of which the above equation becomes

$\frac{\sin ^{-1} \sqrt{k} r_{1}}{\sqrt{k}}=\frac{\delta}{R_{\mathrm{o}} H_{\mathrm{o}}\left(\frac{\Omega_{\mathrm{o}}}{1-\beta}-1\right)^{\frac{1}{2}}} \int_{\alpha_{2}}^{\alpha_{1}} \frac{\mathrm{d} y}{\sqrt{1-y^{2}}}$

where $\delta=\frac{2}{(1-3 \beta)}, \alpha_{1}=\left[1-\left(\frac{1-\beta}{\Omega_{0}}\right)\right]^{\frac{1}{2}}$, and $\alpha_{2}=\alpha_{1}(1+z)^{-\frac{(1-3 \beta)}{2}}$.

The right hand side of the above integral is the same as in (A.1) for $k=1$. Hence, replacing in (A.3) the value of $k$ given by (2) and (7), it is readily seen that

$r_{1}(z)=\frac{\sin \left[\delta \sin ^{-1} \alpha_{1}-\delta \sin ^{-1} \alpha_{2}\right]}{R_{\mathrm{o}} H_{\mathrm{o}}\left(\frac{\Omega_{\mathrm{o}}}{1-\beta}-1\right)^{\frac{1}{2}}}$.

In particular, the limit for a flat universe $\left(\Omega_{0}=1\right)$ yields

$r_{1}(z)=\frac{2}{(1-3 \beta) R_{\mathrm{o}} H_{\mathrm{o}}}\left\{1-(1+z)^{\frac{2}{1-3 \beta}}\right\}$,

which could have been obtained directly from (A.2).

In terms of the deceleration parameter (A.4) may be rewritten as

$r_{1}(z)=\frac{\sin \left[\delta \sin ^{-1} \alpha_{1}-\delta \sin ^{-1} \alpha_{2}\right]}{R_{\mathrm{o}} H_{\mathrm{o}}\left(\frac{2 q_{\mathrm{o}}}{1-3 \beta}-1\right)^{\frac{1}{2}}}$,

which in the limit $\beta \rightarrow 0$ reduces to the usual $\mathrm{CDM}$ result (Weinberg 1972)

$r_{1}(z)=\frac{q_{\mathrm{o}} z+\left(q_{\mathrm{o}}-1\right)\left(\sqrt{2 q_{\mathrm{o}} z+1}-1\right)}{H_{\mathrm{o}} R_{\mathrm{o}} q_{\mathrm{o}}^{2}(1+z)}$.

Equation (A.4), or equivalently (A.6), plays a key role in the derivation of some astrophysical quantities discussed in this paper. 
Acknowledgements. The authors are grateful to the referee, Dr. A. Jaffe, for his many valuable comments. We also acknowledge Dr. Jailson Alcaniz for helpful discussions. This work was partially supported by the project Pronex/FINEP (No. 41.96.0908.00), and CNPq (62.0053/01-1-PADCT III/Milenio).

\section{References}

Alcaniz, J. S., \& Lima, J. A. S. 2001, ApJ, 550, L133

Alcaniz, J. S., \& Lima, J. A. S. 1999, A\&A, 349, 729

Buchalter, et al. 1998, ApJ, 494, 503

Caldwell, R. R., Dave, R., \& Steinhardt, P. J. 1998, Phys. Rev. Lett., 80,1582

Calvão, M. O., Lima, J. A. S., \& Waga, I. 1992, Phys. Lett. A, 162, 233

Carvalho, J. C., Lima, J. A. S., \& Waga, I. 1992, Phys. Rev. D, 46, 2404

Chen, W., \& Wu, Y. S. 1990, Phys. Rev. D, 41, 695

Chiba, T., Sugiyama, N., \& Nakamura, T. 1997, MNRAS, 289, L5

Cooray, A. R., \& Huterer, D. 2000, ApJ, 513, L95

de Bernardis, P., et al. 2000, Nature, 404, 955

Dolgov, A. D. 1997, Lecture presented at the 4th colloque Cosmologie, Paris [astro-ph/9708045]

Efstathiou, G. 1999, MNRAS, 310, 842

Freedman, W. L. 1998, in Proceedings of the 18th Texas Symposium on Relativistic Astrophysics, edited by A. Olinto, J. Frieman, \& D. Schramm (Singapura: World Scientific)

Freese, K., Adams, F. C., Frieman, J. A., \& Mottola, E. 1987, Nucl. Phys. B, 287, 797

Ge, J., Bechtold, J., \& Black, J. H. 1997, ApJ, 474, 67
Gurvits, L. I., Kellermann, K. I., \& Frey, S. 1999, A\&A, 342, 378

Jack, NG. Y. 1992, Int. J. Mod. Phys. D, 1, 145

Kolb, E. W. 1989, ApJ, 344, 543

Kolb, E. W., \& Turner, M. S. 1990, The Early Universe, AddisonWesley, Redwood City

Lima, J. A. S., \& Alcaniz, J. S. 1999, A\&A, 348, 1

Lima, J. A. S., \& Alcaniz, J. S. 2000, A\&A, 357, 393

Lima, J. A. S., \& Maia, J. M. F. 1994, Phys. Rev. D, 49, 5597

Lima, J. A. S., Silva, A. I., \& Viegas, S. M. 2000, MNRAS, 312, 747

Lima, J. A. S., \& Trodden, M. 1996, Phys. Rev. D, 53, 4280

Loh, E., \& Spillar, E. 1986, ApJ, 307, L1

Mattig, W. 1958, Astron. Nachr., 284, 109

Overduin, F. M., \& Cooperstock, F. I. 1998, Phys. Rev. D, 58, 043506

Özer, M., \& Taha, M. O. 1987, Nucl. Phys. B, 287, 776

Perlmutter, S., Aldering, G., Goldhaber, G., et al. 1999, ApJ, 517, 565

Perlmutter, S., et al. 1995, ApJ, 440, L41

Ratra, B., \& Peebles, P. J. E. 1988, Phys. Rev. D, 37, 3406

Riess, A. G., Filippenko, A. V., Challis, P., et al. 1998, AJ, 116, 1009

Sandage, A. 1988, ARA\&A, 26, 561

Sahni, V., \& Starobinsky, A. A. 2000, Int. J. Mod. Phys. D, 9, 373

Songaila, A., Cowie, L. L., Vogt, S., et al. 1994, Nature, 371, 43

Turner, M. S., \& White, M. 1997, Phys. Rev. D, 56, R4439

Turner, M. S. 2000, Phys. Rep., 333, 619

Vishwakarma, R. G. 2000, Class. Q. Grav., 17, 3833

Vishwakarma, R. G. 2001, Class. Q. Grav., 18, 1159

Waga, I. 1993, ApJ, 414, 436

Wang, L., \& Kamionkowski, M. 2000, Phys. Rev. D, 61, 063504

Weinberg, S. 1972, Cosmology and Gravitation (N. Y. John Wiley Sons

Weinberg, S. 1989, Rev. Mod. Phys., 61, 1 